\title{
Type 1 Cardio-Renal Syndrome: A Case Report
}

\author{
Annisa Maloveny \\ Internal Medicine Department \\ Faculty of Medicine and Health Sciences \\ Syarif Hidayatullah State Islamic University \\ Jakarta, Indonesia \\ malovenyanisa@gmail.com
}

\begin{abstract}
The Cardio-Renal Syndrome (CRS) has been defined by Ronco et al. as a pathophysiologic disorder of the heart and kidneys whereby acute or chronic dysfunction of one organ may induce acute or chronic dysfunction of the other. A 55-year old woman was brought to the emergency room presented with acute worsening of heart failure. There were concomitant diseases diabetes mellitus, lung infection and anemia with hypotension and metabolic acidosis episodes. Patient became oligouric with worsening renal function which was resistant to diuretic therapy.
\end{abstract}

Keywords - Cardio-Renal Syndrome; heart failure; oliguric; renal function; diabetes mellitus

\section{INTRODUCTION}

The Cardio-Renal Syndrome (CRS) has been defined by Ronco et al. as a pathophysiologic disorder of the heart and kidneys whereby acute or chronic dysfunction of one organ may induce acute or chronic dysfunction of the other. The Cardio-Renal Syndromes are subdivided into five different subtypes in order to allow physicians to better understand the situation and offer adequate treatment. The type $1 \mathrm{CRS}$ is characterized by acute worsening of heart failure leading to acute kidney injury (AKI). It is considered to be the most common type of CRS and is present in approximately $25 \%$ of patients admitted with acute decompensated heart failure (ADHF) [1,2]

\section{CASE PRESENTATION}

A 55-year old woman was brought to the emergency room presented with worsening shortness of breath. Her past medical history was relevant for hypertension, diabetes mellitus, dyslipidemia, coronary artery disease. She also had history of kidney disease but was never advised to have hemodialysis. She had lack of compliance visiting the doctor for her condition. One week before admission to the hospital, she had complaint of productive cough.

On admission, the patient's physical examination revealed severe difficulty in breathing (dyspnea), a blood pressure of $140 / 90 \mathrm{mmHg}$, jugular venous distention, decreased bilateral basal breath sounds, no fever and no leg edema. She was not obese. Initial abnormal serum laboratory results included: Hemoglobin $6.4 \mathrm{~g} / \mathrm{dl}$, Hematocrit 23\%, white blood cell
(WBC) count of 11400/mm3, thrombocyte 331000/mm3, creatinine of $3.3 \mathrm{mg} / \mathrm{dl}$ ureum $120 \mathrm{mg} / \mathrm{dl}$, Random blood sugar $285 \mathrm{mg} / \mathrm{dl}$. She had mild hyponatremia and hyperkalemia (potassium $5.1 \mathrm{mmol} / \mathrm{l}$, sodium $129 \mathrm{mmol} / \mathrm{l}$, chloride $95 \mathrm{mmol} / \mathrm{l}$, bicarbonate $23 \mathrm{mmol} / \mathrm{l}$ ). Further investigations revealed severe cardiomegaly pulmonary edema with bilateral effusion on chest X-ray. Pulseoxymeter showed $\mathrm{SpO}_{2}$ 99\%. Electrocardiogram (ECG) on admission showed a sinus tachycardia.

The patient was admitted to the intensive care unit (ICU) with acute decompensated of $\mathrm{CHF}$ (congestive heart failure). Intravenous furosemid was given due to fluid overload with dose titration started from 40 until $80 \mathrm{mg}$ daily. ACE inhibitors (captopril) was given $25 \mathrm{mg}$ twice daily. Empiric antibiotics were started combining ceftriaxone and levofloxacin. Insulin was given to reduce blood sugar level. Transfusion $200 \mathrm{~cm} 3$ or packed red cell was given with extra caution due to fluid overload condition. Sputum cultures could not be conducted due to unavailability of sputum collection. On day 3 of admission, the patient became unconscious and hypotension (blood pressure $85 / 65 \mathrm{mmHg}$ ) and developed severe metabolic acidosis with $\mathrm{ABG}$ showing: $\mathrm{pH} 7.14, \mathrm{PaCO}_{2} 28 \mathrm{mmHg}, \mathrm{PaO}_{2}$ $75 \mathrm{mmHg}, \mathrm{HCO}_{3}{ }^{-} 10 \mathrm{mmol} / \mathrm{l}$ and she was intubated. She concomitantly became oligouric and serum creatinine started to increase (from $3.3 \mathrm{mg} / \mathrm{dl}$ to $5.3 \mathrm{mg} / \mathrm{dl}$ ) and urine output decrease. She received sodium bicarbonate whenever her bicarbonate level was less than 15 . She was on intravenous vasopressor (dobutamine and norepinephrine titration) until achieve the mean arterial pressure target. After her blood pressure became stabile (normotensive), She received continuous furosemide infusion at $10 \mathrm{mg}$ per hour to increase the urine output. However she remained oligouric even after 24 hours of therapy. She continued process of the renal replacement therapy with referral to hemodialysis center due to resistant to intravenous diuretic therapy (furosemid).

\section{DISCUSSION}

Our patient can be classified in the type 1 CRS. Type 1 CRS occurs in about $25 \%$ of patients hospitalized for acute decompensated heart failure (ADHF). Among these patients, underlying chronic kidney disease (CKD) is quite common and contributes to acute kidney injury (AKI) in $60 \%$ of all cases studied. AKI is an independent mortality risk factor in 
acute decompensated heart failure patients, including those with reduced left ventricular ejection fraction [2]. Her chronic heart failure with aggravation of acutely decompensated heart failure (ADHF) led to AKI on the top of a probable chronic diabetic nephropathy $[2,3]$.

AKI in our patient was diagnosed using the definition of RIFLE, AKIN and KDIGO classifications as an increase in serum creatinine to more than 1.5 -fold over baseline. The factors usually implicated in the pathogenesis of AKI in type 1 CRS are multiple and complex. But the main classical pathophysiological mechanism to consider first in any type 1 CRS remains the hemodynamic factor, an inadequate renal perfusion secondary to an acute low cardiac output (CO) or acute heart failure (HF) $[2,3]$.

In the classification of type $1 \mathrm{CRS}$, severe cardiomegaly with left ventricular (LV) pump failure as a possible etiology for low CO. Patients with ADHF and type 1 CRS have usually poor outcomes especially when accompanied by severe hypotension that is not reversible. Patients with HF due to left ventricular (LV) pump failure have reductions in $\mathrm{CO}$ and stroke volume. The fact that our patient had a probable low LVEF due to severe dilated cardiomyopathy contributed to her hemodynamic instability once the worsened heart failure occurred. Apart from low renal blood flow from reduced cardiac index and various neurohumoral mechanism, volume overload in the venous compartment causing increased renal venous pressure in an important contributor of reduced glomerular filtration. Diuretic therapy was resistant for this patient which is common in patients with CHF. After the hypotension period, she was given dobutamine therapy. Dobutamine therapy can be used to temporarily improve hemodynamics in type-1 CRS by improving cardiac index and reducing renal venous congestion by augmenting RV function. Dobutamine therapy can be tried in patients with acute CRS refractory to diuretic therapy. Patients whose resistance to diuretic therapy is due to low cardiac output may require inotropic therapy, and other options (eg. ultrafiltration and vasopressin antagonists) [1,2,3].

Hemodynamic mechanisms play a major role in type 1 CRS in presence of ADHF leading to decreased renal arterial flow and a consequent fall in glomerular filtration rate (GFR). Once hemodynamics have been restored, renal and cardiac parameters come back to normal. Different hemodynamic profiles have been proposed: in "cold" pattern patients, reduction in effective circulation fluid volume (ECFV) represents the main hemodynamic change, while there is a marked increase in central venous pressure (CVP) in "wet" pattern patients. "Cold" patients also present with decrease in renal blood flow related to the renin angiotensin-aldosterone system (RAAS) and systemic nervous system activation causing afferent vasoconstriction, decreased renal blood flow and decreased effective glomerular perfusion pressure. Patients who present with a "wet" hemodynamic profile display increased pulmonary and/or systemic congestion. In these patients, high CVP directly affects renal vein and kidney perfusion pressure. Central venous pressure increase also results in increased interstitial pressure with tubular collapse and progressive decline in GFR. "Warm and wet" patients represent the most frequent profile in acute and chronic advanced heart failure. Mechanisms of increased CVP are quite similar to "cold" profile patients, but renal perfusion pressure is less affected because of higher arterial blood pressures. Non-hemodynamic mechanisms were also proposed as involved in type 1 CRS including sympathetic nervous system (SNS) and renin-angiotensin aldosterone system (RAAS) activation, chronic inflammation and imbalance in the proportion of reactive oxygen species (ROS)/nitric oxide (NO) production. Patients with ADHF show more frequently defective regulation of monocyte apoptosis and activation of inflammatory pathways compared with healthy subjects $[4,5]$.

\section{CONCLUSION}

As a summary, acute lowering of cardiac output secondary to pumping failure can lead to renal hypoperfusion. Pumping failure is a cause of decreased cardiac output and can lead to hemodynamic instability if superimposed on preexisting heart failure, even in well preserved systolic blood pressure cases. This hemodynamic instability may cause simultaneously oligo anuria and renal injury.

\section{REFERENCES}

[1] C. Ronco, M. Haapio, A.A. House, N. Anavekar, R. Bellomo. "Cardiorenal syndrome," J Am Coll Cardiol, vol. 52, no. 19, pp. 1527-39, Nov 2008.

[2] House AA, Anand I, Bellomo R, Cruz D, Bobek I, Anker SD, Aspromonte N, Bagshaw S, Berl T, Daliento L, Davenport A, Haapio M, Hillege H, McCullough P, Katz N, Maisel A, Mankad S, Zanco P, Mebazaa A, Palazzuoli A, Ronco F, Shaw A, Sheinfeld G, Soni S, Vescovo G, Zamperetti N, Ponikowski P, Ronco C. "Definition and classification of Cardio-Renal Syndromes: workgroup statements from the 7th ADQI Consensus Conference," Nephrol Dial Transplant, vol. 25, no. 5, pp. 1416-20, May 2010.

[3] Hata N, Yokoyama S, Shinada T, et al. "Acute kidney injury and outcomes in acute decompensated heart failure: evaluation of the RIFLE criteria in an acutely ill heart failure population," Eur J Heart Fail, vol. 12, no. 1, pp. 32-7, Jan 2010.

[4] Prins KW, Wille KM, Tallaj JA, Tolwani AJ. "Assessing continuous renal replacement therapy as a rescue strategy in cardiorenal syndrome 1," Clin Kidney J, vol. 8, no. 1, pp. 87-92, Feb 2015.

[5] Di Lullo L, Bellasib A, Barberaa V, Russoc D, Russoc R, Di Ioriod B, Cozzolinoe M, Roncof C. "Pathophysiology of the cardio-renal syndromes types 1-5: An up to date," Indian Heart J, vol. 69, no. 2, pp. 255 65, Mar-Apr 2017. 